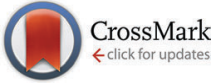

Cite this: Phys. Chem. Chem. Phys., 2015, 17, 22438

Received 28th April 2015 Accepted 21st July 2015

DOI: $10.1039 / c 5 c p 02472 b$

www.rsc.org/pccp

\title{
Molecular dynamics simulations and NMR spectroscopy studies of trehalose-lipid bilayer systems
}

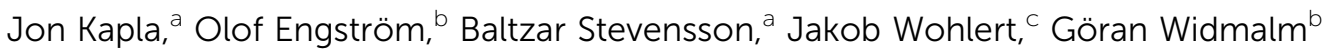 \\ and Arnold Maliniak*a
}

\begin{abstract}
The disaccharide trehalose (TRH) strongly affects the physical properties of lipid bilayers. We investigate interactions between lipid membranes formed by 1,2-dimyristoyl-sn-glycero-3-phosphocholine (DMPC) and TRH using NMR spectroscopy and molecular dynamics (MD) computer simulations. We compare dipolar couplings derived from DMPC/TRH trajectories with those determined (i) experimentally in TRH using conventional high-resolution NMR in a weakly ordered solvent (bicelles), and (ii) by solid-state NMR in multilamellar vesicles (MLV) formed by DMPC. Analysis of the experimental and MD-derived couplings in DMPC indicated that the force field used in the simulations reasonably well describes the experimental results with the exception for the glycerol fragment that exhibits significant deviations. The signs of dipolar couplings, not available from the experiments on highly ordered systems, were determined from the trajectory analysis. The crucial step in the analysis of residual dipolar couplings (RDCs) in TRH determined in a bicelle-environment was access to the conformational distributions derived from the MD trajectory. Furthermore, the conformational behavior of $\mathrm{TRH}$, investigated by $J$-couplings, in the ordered and isotropic phases is essentially identical, indicating that the general assumptions in the analyses of RDCs are well founded.
\end{abstract}

\section{Introduction}

The bioprotective properties of saccharides in general and trehalose $(\mathrm{TRH})$ in particular have motivated significant attention in the scientific literature. ${ }^{1-5}$ These properties have stabilizing effects on biological membranes under extreme thermal and mechanical conditions, which are particularly important during the process of dehydration. ${ }^{6}$ Although the detailed mechanism of trehalosemembrane stabilization is not yet clarified, the effect of preserving the cell structure and biological function is well known.

Several approaches for the molecular description of membranesugar interactions have been suggested: (a) a preferential exclusion model, where sugars are excluded from the vicinity of the lipid membrane, thus preserving the natural hydration shell of the bilayer, ${ }^{1,6-9}$ (b) the water-entrapment hypothesis, resting on the assumption that the sugars interact strongly with both lipids and water, which increases favorable hydration near the membrane, ${ }^{10-15}$

\footnotetext{
${ }^{a}$ Department of Materials and Environmental Chemistry, Arrhenius Laboratory,

Stockholm University, SE-106 91 Stockholm, Sweden.

E-mail: arnold.maliniak@mmk.su.se

${ }^{b}$ Department of Organic Chemistry, Arrhenius Laboratory, Stockholm University,

SE-106 91 Stockholm, Sweden

${ }^{c}$ Wallenberg Wood Science Center, Royal Institute of Technology,

SE-100 44 Stockholm, Sweden
}

and the vitrification hypothesis, where a glassy sugar-water matrix that protects the membrane from mechanical stress is formed. ${ }^{16-20}$ There are, however, clear indications that the explanation may consist of a combination of several hypotheses. Two key concepts relevant for these studies are the hydration process ${ }^{21-24}$ and the conformational behavior of trehalose. ${ }^{25-27}$

Many experimental and theoretical approaches have been employed to study membrane-sugar interactions. In particular, computer simulation is a powerful tool for investigations of the detailed picture of complex biological systems, and several studies of lipid bilayer-trehalose interactions have been reported using different degrees of sophistication for the interaction model. ${ }^{22,28-36}$

Recently, ${ }^{5}$ we carried out molecular dynamics (MD) computer simulations for analysis of interactions between a 1,2-dimyristoylsn-glycero-3-phosphocholine (DMPC) bilayer and trehalose (see Fig. 1). Using MD simulations and a simple two-state interaction model we were able to confirm the hypotheses indicated above, suggested for the explanation of the membrane-sugar interactions. We showed, by calculating the net affinity of TRH for the DMPC bilayer, that the concept of attraction (low TRH content) and exclusion (high TRH content) of sugars from the membrane can be used in fully hydrated as well as in dehydrated membranes. Furthermore, the replacement of water by trehalose molecules was observed at the bilayer interface. The compressibility modulus and 

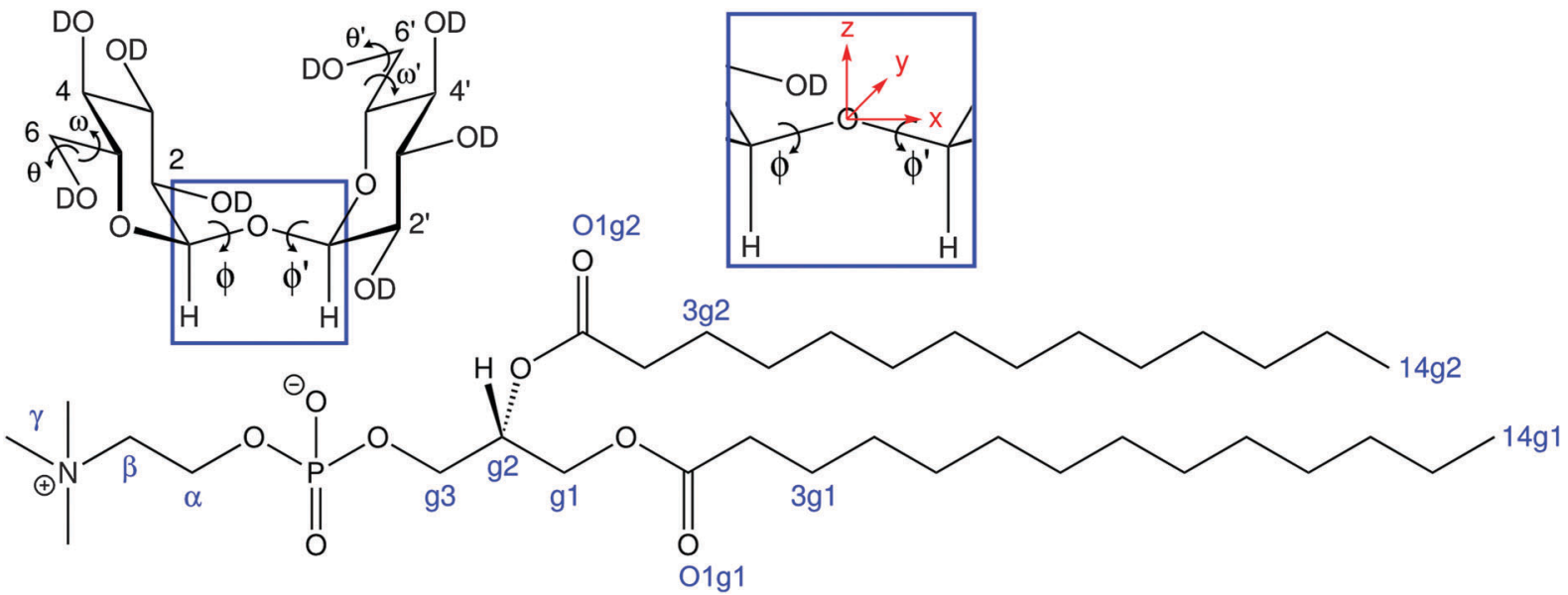

Fig. 1 Schematic structures of 1,2-dimyristoyl-sn-glycero-3-phosphocholine (DMPC) lipid and trehalose (TRH) molecules with the relevant coordinate system and dihedral angles.

increased order, manifested by a drastic reduction in the lateral diffusion were attributed to the formation of a glassy state upon increased TRH content.

In the present work we have carried out measurements of NMR spin-spin interactions on trehalose in isotropic and ordered systems. In the isotropic liquid only the $J$-couplings are observable, whereas the direct dipolar interactions are averaged (by the rapid molecular tumbling) to zero. In the anisotropic systems, on the other hand, direct dipole-dipole interactions can be observed and provide valuable information on the molecular structure and the orientational order. In principle, there are two NMR spectroscopy techniques to study anisotropic dipolar interactions: (a) conventional high-resolution NMR where a weakly ordered solvent ${ }^{37-39}$ is used, and (b) solid-state NMR. ${ }^{40-43}$

Weakly ordered anisotropic media, used for measurements of dipolar interactions (frequently referred to as residual dipolar couplings or RDCs), consist of dilute bilayer-like assemblies (bicelles) formed by various lipids, e.g. DMPC and DHPC. For carbohydrates, RDCs provide important information about the complex conformational processes taking place in solution. ${ }^{44-51}$

We have used trajectories generated in the MD simulations carried out in the previous study ${ }^{5}$ for interpretation of experimental herein acquired NMR parameters and analyses of the membrane-TRH interactions. A combination of MD computer simulations and NMR spectroscopy is a powerful tool for investigations of the detailed molecular picture of complex chemical systems, since all possible dipolar interactions can readily be calculated from an MD trajectory.

\section{Methods}

\section{Molecular dynamics simulations}

The trajectories used in the analyses were generated in our previous investigation, focused on the interactions of trehalose and the DMPC bilayer. $^{5}$

The MD computer simulation study was carried out with a lipid bilayer consisting of 128 DMPC molecules with ten different trehalose concentrations: 0-260 TRH molecules (labelled as TRHN, where $N$ is the total number of TRH molecules), corresponding to a maximum trehalose content of $w_{\mathrm{TRH}}=0.20$ (wt/wt). The water content in all the simulations ranged between 10000 and 14000 molecules. Only the most essential details of the methodological aspects are repeated here. The lipid interactions were modeled using the CHARMM-based and AMBER-compatible force field SLIPIDS (Stockholm Lipids). ${ }^{52}$ The TRH interaction parameters were GLYCAM06-based ${ }^{53}$ and AMBER-compatible. The water interactions were described using the TIP3P model. ${ }^{54}$ Both AMBER and SLIPIDS force fields were parameterized employing the TIP3P water model. Whereas the dynamics of TIP3P water is known to be too fast, the equilibrium properties are in good agreement with experimental results. ${ }^{5,56}$ In particular, the $\mathrm{O}-\mathrm{O}$ radial distribution function $\left(g_{\mathrm{O}-\mathrm{O}}\right)$ and the heat of vaporization are correctly predicted indicating that hydrogen bonds formed by water are reasonably well described, even though the kinetics is not. The simulations were carried out in the GROMACS v4.5.5 program suite ${ }^{57}$ in the NPT ensemble at a temperature of $310 \mathrm{~K}$ and a pressure of $100 \mathrm{kPa}$. All electrostatics were treated with the Particle Mesh Ewald (PME) summation method. ${ }^{58}$ The simulations were subject to $100 \mathrm{~ns}$ of equilibration, after which 100-140 ns were used for analysis. The MD simulations of trehalose in the isotropic aqueous solutions were carried out in three different samples with 10 , 50 and 100 TRH dissolved in 5000 water molecules, corresponding to $0.1,0.5$ and $1.0 \mathrm{M}$ solutions.

\section{Experimental}

\section{NMR spectroscopy}

The disaccharide $\alpha, \alpha$-trehalose ( $\alpha$-D-Glucopyranosyl $\alpha$-D-Glucopyranoside) was obtained from Nutritional Biochemicals Corporation (Cleveland, Ohio, USA) and the lipids 1,2-dihexanoylsn-glycero-3-phosphocholine (DHPC) and 1,2-dimyristoyl-sn-glycero3-phosphocholine (DMPC) were purchased from Avanti Lipids (Alabaster, Alabama, USA). The lipid bicelle sample was prepared by dissolving DHPC (33 mM) and DMPC $(94 \mathrm{mM})$ in $\mathrm{D}_{2} \mathrm{O}$ (lipid ratio 1:2.8; lipid concentration $8 \% \mathrm{w} / \mathrm{v}$ ). The preparation 
was vibro-mixed for $5 \mathrm{~min}$, heated to $38{ }^{\circ} \mathrm{C}$ for $30 \mathrm{~min}$ and then cooled to $5{ }^{\circ} \mathrm{C}$ for $30 \mathrm{~min}$. The procedure was repeated three times. The cooled mixture $(0.55 \mathrm{~mL})$ was added to an NMR tube containing $9.4 \mathrm{mg}$ of TRH that had been freeze-dried from $\mathrm{D}_{2} \mathrm{O}$, resulting in a $\sim 50 \mathrm{mM}$ solution. Measurements in the isotropic phase were performed on a TRH sample $(106 \mathrm{mM})$ in $\mathrm{D}_{2} \mathrm{O}$ with trimethylsilyl-2,2,3,3-tetradeuteropropionic acid (TSP, $1 \mathrm{mM}$ ) as the chemical shift reference $\left(\delta_{\mathrm{H}}=0.0\right)$. Resonance assignments were facilitated by chemical shift data available from the literature. ${ }^{59}$ NMR experiments were recorded on a $600 \mathrm{MHz}$ Bruker AVANCE III spectrometer equipped with a $5 \mathrm{~mm}$ inverse Z-gradient TXI $\left({ }^{1} \mathrm{H} /{ }^{13} \mathrm{C} /{ }^{31} \mathrm{P}\right)$ probe at $38{ }^{\circ} \mathrm{C}$, calibrated with a methanol- $d_{4}$ sample. ${ }^{60}$ The anisotropy of the system was monitored by measurements of the ${ }^{2} \mathrm{H}$ quadrupolar coupling of $\mathrm{D}_{2} \mathrm{O}$, ranging between $13.7 \mathrm{~Hz}$ and $14.0 \mathrm{~Hz}$ as determined prior to and after the experiments had been performed on the sample.

The J-HMBC experiments ${ }^{61}$ were acquired with 32 scans and with scaling factors $\kappa$ varying between 21 and 27 . The number of points in the $F_{2}$ and $F_{1}$ dimensions were 8k and 512, respectively; carrier frequencies were set at $3.0 \mathrm{ppm}$ and $55 \mathrm{ppm}$, respectively, and the spectral widths were set to $6 \mathrm{ppm}$ and $100 \mathrm{ppm}$, respectively. The free induction decays (FIDs) were zero-filled to $16 \mathrm{k}$ in the indirect dimension and linear prediction using 64 coefficients and 512 points were applied to the indirect dimension prior Fourier transformation with magnitude processing in the direct dimension. Two- and three-bond heteronuclear couplings were extracted from projections in the indirect dimension at different ${ }^{1} \mathrm{H}$ chemical shifts. The measured couplings were divided by the corresponding scaling factor and averages from four different experiments were calculated.

The ${ }^{1} \mathrm{H},{ }^{13} \mathrm{C}-\mathrm{HSQC}-\mathrm{HECADE}$ experiments ${ }^{62,63}$ were recorded with 16k and 512 data points in the $\mathrm{F}_{2}$ and $\mathrm{F}_{1}$ dimensions, respectively, and the carrier frequencies were set at $3.0 \mathrm{ppm}$ and $60 \mathrm{ppm}$, respectively, using 16 scans. The duration of the TOCSY mixing time was $80 \mathrm{~ms}$ and the scaling factor of the ${ }^{1} J_{\mathrm{CH}}$ splitting in the indirect dimension was set to 0.8 . The FIDs were zero-filled once in each dimension and an exponential window function of $0.3 \mathrm{~Hz}$ and a cosine window function were applied to the $F_{2}$ and $F_{1}$ dimensions, respectively, prior to Fourier transformation. One-, two- and three-bond heteronuclear couplings were extracted from the differences in the peak-position along the $\mathrm{F}_{2}$ dimension of the doublet components $\left(\mathrm{F}_{1}\right.$ dimension, E.COSY-type cross-peak) and the sign of the couplings was determined from the cross-peak tilt relative to the one-bond couplings that were assumed to be positive.

The ${ }^{1} \mathrm{H},{ }^{13} \mathrm{C}$-CT-CE-HSQC experiments ${ }^{64}$ were performed with 64 scans and the number of points in the $F_{2}$ and $F_{1}$ dimensions were $1 \mathrm{k}$ and 512, respectively; carrier frequencies were set at $3.2 \mathrm{ppm}$ and $77 \mathrm{ppm}$ employing spectral widths of $7 \mathrm{ppm}$ and $165 \mathrm{ppm}$. The FIDs were zero-filled to $16 \mathrm{k}$ in the indirect dimension and linear predictions using 36 coefficients and 512 points were applied to the indirect dimension prior Fourier transformation. One-bond heteronuclear couplings, scaled by a factor of 2.0, were extracted from projections of the indirect dimension at different ${ }^{1} \mathrm{H}$ chemical shifts.

The phase-sensitive ${ }^{1} \mathrm{H},{ }^{1} \mathrm{H}$-DQF-COSY ${ }^{65}$ experiments were performed using 8 scans. The carrier was set to $4.65 \mathrm{ppm}$ and the spectral widths were $4 \mathrm{ppm}$ in both dimensions. The number of recorded data points in the $F_{2}$ and $F_{1}$ dimensions was $32 \mathrm{k}$ and 128 , respectively; zero-filling was carried out to $256 \mathrm{k}$ and 512 points prior Fourier transformation. Resolution enhancement was achieved in the direct dimension by applying a Lorentzian-to-Gaussian window function $(\mathrm{lb}=-0.5 \mathrm{~Hz}$ and $\mathrm{gb}=0.2$ ) to the FIDs. Vicinal ${ }^{1} \mathrm{H},{ }^{1} \mathrm{H}$ couplings were extracted by the analysis of multiplet patterns of cross-peak slices taken in the direct dimension using the J-doubling methodology ${ }^{66}$ employing an in-house written Matlab script.

\section{Results and discussion}

\section{NMR dipole-dipole interactions}

Dipolar couplings in DMPC. We start the analysis by considering the ${ }^{1} \mathrm{H}^{13} \mathrm{C}$ dipolar couplings in DMPC that were determined in our previous solid-state experimental study ${ }^{67}$ of lipid bilayers. The information contained in these couplings is identical (in the fast limit of the NMR time scale) to the quadrupolar interaction determined from deuterium NMR spectra. The ${ }^{1} \mathrm{H}^{13} \mathrm{C}$ dipolar coupling (in $\mathrm{Hz}$ ) can be calculated from the MD trajectory using

$$
d_{i j}=-\frac{\mu_{0} \gamma_{i} \gamma_{j} \hbar}{8 \pi^{2}}\left\langle\frac{1}{2}\left(3 \cos ^{2} \theta_{i j}-1\right) r_{i j}{ }^{-3}\right\rangle_{\theta}
$$

where $\theta_{i j}$ is the angle between the spin-spin vector and the bilayer normal, $r_{i j}$ is the spin-spin distance, and the other symbols have their usual meaning. In our MD simulations the bond lengths were constrained using the $\operatorname{LINCS}^{68,69}$ algorithm, therefore the $\mathrm{C}-\mathrm{H}$ bonds had an $r_{\mathrm{CH}}=109 \mathrm{pm}$ (as in the force field), which corresponds to the vibrationally averaged $\mathrm{C}-\mathrm{H}$ distance in methylene groups. ${ }^{70}$ The angular bracket denotes an average over all molecular orientations and corresponds to $S_{\mathrm{CH}} \cdot r^{-3}$, where $S_{\mathrm{CH}}$ is the standard order parameter used for characterization of the local order in membranes. We assume that the bilayer normal and the $z$-axis of the simulation box coincide. In analogy with deuterium quadrupolar splittings, the experimental dipolar couplings provide information on the magnitude, but not on the sign of the interaction. In contrast, both the magnitude and sign are obtained for the dipolar couplings calculated from the MD trajectory. Thus, the sign of the experimental couplings can be derived for all the interactions by a combination of information contained in experimental and calculated dipolar couplings. In Fig. 2 the experimental and the trajectory calculated (using eqn (1)) dipolar couplings are displayed for the different fragments of DMPC in the TRH0 sample, which does not contain any trehalose. Clearly, the signs of all the couplings can be predicted using the values calculated from the trajectory. The only exceptions are the dipolar couplings of the g2 and g3 carbons where the simulated values significantly deviate from the experiments and prevent the determination of the sign(s). This deviation can be ascribed to an imperfection of the force field used in the present MD simulation, which has in fact recently been pointed out. ${ }^{71}$ In addition, the motion of these fragments is considerably slower than other conformational transitions, which limits a proper averaging, resulting in large 


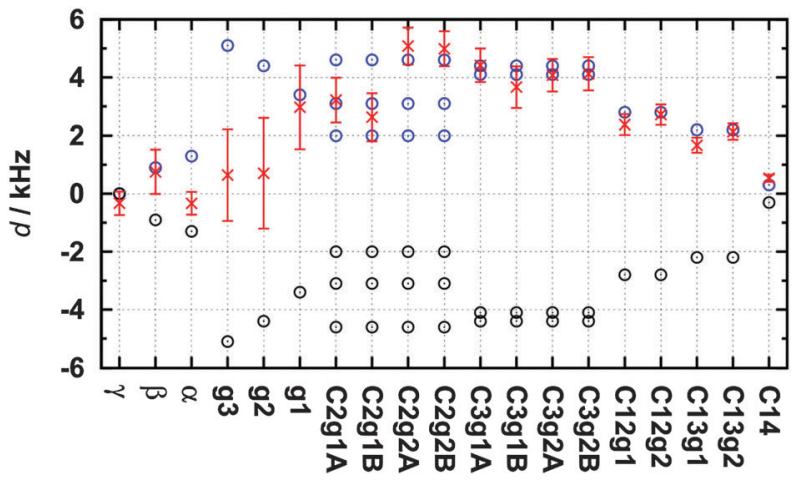

Fig. 2 NMR dipolar couplings in DMPC: the negative and positive values of the experimental dipolar couplings correspond to black and blue circles, respectively. The values derived from the trajectory are represented by red crosses with associated error bars (corresponding to $1 \mathrm{SD}$ ). In principle, the different fragments of the alkyl chains can correspond to four different dipolar interactions. In the experimental study, ${ }^{42}$ we were at most able to determine three couplings in one fragment $(\mathrm{C} 2 \mathrm{~g} 1$ and $\mathrm{C} 2 \mathrm{~g} 2)$. Thus, the individual couplings ( $\mathrm{A}$ and $\mathrm{B}$ ) in the methylene group were assigned based on the best agreement between calculated and experimental values.

error bars. This dynamics can be evaluated by considering the orientational time correlation functions (TCFs) of $\mathrm{C}-\mathrm{H}$ vectors corresponding to the NMR dipolar interactions. The TCF is defined as ${ }^{72} C(t)=\left\langle D_{00}{ }^{2}(\Omega(0)) \cdot D_{00}{ }^{2}(\Omega(t))\right\rangle$, where $D_{00}{ }^{2}(\Omega)$ is a second rank Wigner rotation matrix element, and $\Omega$ represents time dependent Euler angles relating the orientation of the bilayer normal to a vector fixed in the lipid. In Fig. 3 the TCFs of $\mathrm{C}-\mathrm{H}$ vectors are shown for methylene/methine groups, which are denoted $\alpha, \beta, \mathrm{g}_{1}, \mathrm{~g}_{2}$ and $\mathrm{g}_{3}$ ( $c f$. Fig. 1 ). The dynamics of the $\mathrm{C}-\mathrm{H}$ vectors in the choline residue ( $\alpha$ and $\beta$ methylene groups) is significantly faster compared to those in the glycerol residue $\left(\mathrm{g}_{1}, \mathrm{~g}_{2}\right.$ and $\left.\mathrm{g}_{3}\right)$, which is also reflected in the larger error bars related to the dipolar couplings (Fig. 2) of the latter. It can also be noted that all the TCFs decay nearly to zero at longer times. In fact, the long time limit of these TCFs is $\lim _{t \rightarrow \infty} C(t)=S^{2}$, where $S^{2}$ is the orientational order parameter of the vector. Clearly, the order parameters of the $\mathrm{C}-\mathrm{H}$ vectors in the $\mathrm{g}_{2}$ and $\mathrm{g}_{3}$ groups are too low, which is also reflected in a too small magnitude of the simulated dipolar couplings.

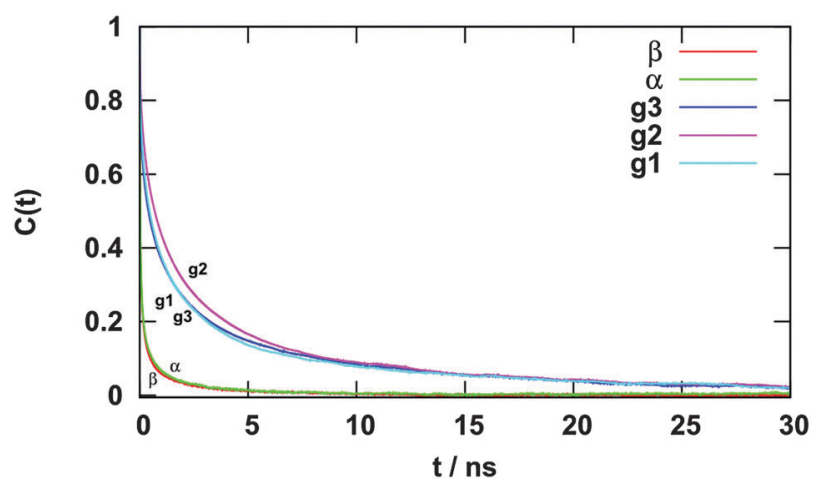

Fig. 3 Normalized time correlation functions (TCFs) calculated for various $\mathrm{C}-\mathrm{H}$ vectors in DMPC: $\beta$ (red), $\alpha$ (green), $\mathrm{g}_{3}$ (blue), $\mathrm{g}_{2}$ (magenta), and $\mathrm{g}_{1}$ (cyan).
Dipolar couplings in TRH. Experimental RDCs can be obtained from NMR spectroscopy experiments designed to extract $J$ couplings. By performing experiments on two samples, one containing the analyte in a weakly ordered medium (viz. a lyotropic liquid crystalline phase consisting of DMPC/DHPC bicelles) and one isotropic sample (the analyte in buffer solution only), RDCs can be calculated as $d_{i j}=\left(\Delta_{i j}-J_{i j}\right) / 2$, where $J_{i j}$ is the isotopic scalar coupling and $\Delta_{i j}$ is the corresponding apparent peak-splitting in the spectrum ${ }^{73}$ in the anisotropic phase. When $J_{i j} \gg\left|d_{i j}\right|$ the sign of the dipolar coupling can be determined readily.

Homonuclear ${ }^{1} \mathrm{H},{ }^{1} \mathrm{H}$ couplings can be measured from the cross-peak patterns in a phase-sensitive DQF-COSY spectrum ${ }^{74}$ where the active coupling appears as an anti-phase doublet and passive couplings appear in-phase. Small couplings are difficult to extract accurately due to the spectral overlap of the multiplet components; however, these $J$ values can be extracted by utilizing the $J$-doubling methodology, ${ }^{66}$ which is based on multiplet deconvolution. One-bond heteronuclear couplings can readily be measured in a ${ }^{1} \mathrm{H},{ }^{13} \mathrm{C}-\mathrm{CT}$-CE-HSQC spectrum, ${ }^{64}$ as half of the cross-peak splitting in the indirect dimension (Fig. 4). The scaling of the coupling by a factor of two increases the accuracy of the measurement. Two- and three-bond heteronuclear couplings can be accessed from a set of $\mathrm{J}^{-} \mathrm{HMBC}^{61}$ experiments. The couplings are measured in the indirect dimension and the problem with spectral overlap can be limited by varying the scaling factor, which also improves the accuracy of the measurements. An advantage of

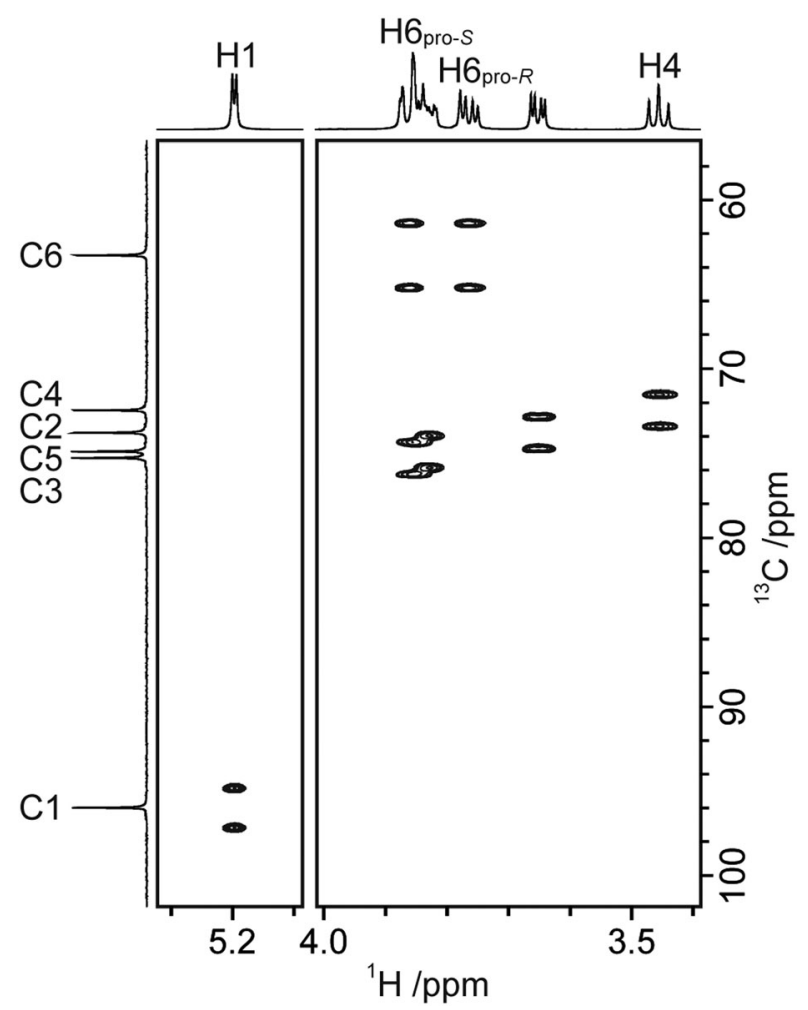

Fig. $4{ }^{1} \mathrm{H}_{,}^{13} \mathrm{C}-\mathrm{CT}-\mathrm{CE}-\mathrm{HSQC}$ NMR spectrum for measuring one-bond heteronuclear couplings of TRH in anisotropic DMPC/DHPC bicelle medium. The $J+2 d$ values are determined from the peak separation in the $F_{1}$ dimension $\left({ }^{13} \mathrm{C}\right.$ dimension). 
Table 1 NMR couplings for spin-pairs measured in the anisotropic ( $\Delta$ ) and isotropic (J) phases that were used to calculate the RDCs (d)

\begin{tabular}{lrrr}
\hline Spin-pairs & \multicolumn{1}{c}{$J$} & \multicolumn{1}{c}{$d^{a}$} \\
\hline H1-H2 & 2.93 & 3.89 & -0.48 \\
H2-H3 & 10.65 & 9.90 & 0.37 \\
H3-H4 & 8.87 & 9.11 & -0.12 \\
H4-H5 & 8.87 & 9.96 & -0.55 \\
H6pro- - -H5 & 4.78 & 5.25 & -0.24 \\
H6pro-S-H6pro- $R$ & -9.09 & -12.08 & 1.50 \\
C1-H1 & 175.85 & 172.65 & 1.60 \\
C2-H2 & 142.50 & 143.82 & -0.66 \\
C4-H4 & 142.70 & 144.36 & -0.83 \\
C6-H6pro-S & 144.78 & 144.53 & 0.13 \\
C1-H1 & 3.18 & 3.31 & -0.06 \\
C1-H2 & -1.32 & -0.82 & -0.25 \\
C1-H3 & 1.26 & 0.74 & 0.26 \\
C2-H1 & -1.25 & -1.42 & 0.09 \\
C2-H4 & 1.34 & 1.30 & -0.02 \\
C3-H1 & 5.44 & 5.51 & -0.03 \\
C3-H2 & -4.19 & -4.80 & 0.30 \\
C3-H4 & -4.43 & -4.48 & 0.03 \\
C4-H2 & 1.38 & 1.30 & 0.04 \\
C4-H3 & -4.55 & -4.63 & 0.04 \\
C4-H6pro- $R$ & 1.36 & 1.37 & 0.00 \\
C5-H4 & -4.52 & -3.99 & -0.26 \\
C5-H6pro- $R$ & -1.93 & -1.83 & -0.05 \\
C6-H4 & 3.72 & 3.70 & 0.01 \\
$a$ Estimated errors are \pm 0.2 Hz. & & \\
& & &
\end{tabular}

the J-HMBC experiment is that one can determine $J$ couplings across the glycosidic linkage. Single- and multiple-bond heteronuclear couplings can also be accessed by the ${ }^{1} \mathrm{H},{ }^{13} \mathrm{C}-\mathrm{HSQC}-$ HECADE $^{62,63}$ experiment, in which the coupling is measured from a splitting in the direct dimension. The experiment additionally yields information about the relative sign of the coupling.

These NMR experiments were used to generate a data-set of experimentally determined RDCs for trehalose dissolved in the lyotropic liquid crystalline phase consisting of DMPC/DHPC bicelles (Table 1). The different experiments complement each other not only because different types of specific information can be extracted from them (e.g. $J$ couplings of one-, two- and three-bond interactions, relative sign, transglycosidic $J$ couplings) but also due to the fact that the same coupling can be measured in different dimensions thus increasing the reliability of the measurements using two methods. The number of linearly independent dipolar vectors in TRH is reduced, compared to other disaccharides, due to the $C_{2}$ symmetry of TRH which makes the disaccharide appear as a monosaccharide in the NMR spectrum. The spatial arrangement of atoms in the adopted ${ }^{4} C_{1}$ chair conformation of the glucose residues results in several parallel dipolar vectors, which also limits the number of independent RDCs in comparison to other hexose epimers. The magnitude of the experimental RDCs seems to be low relative to similar systems. ${ }^{49,75}$ In earlier studies of oligosaccharides based on RDC analysis we successfully used DMPC/DHPC lipid concentrations of $8-10 \%(\mathrm{w} / \mathrm{v})^{76,77}$ and the study of sucrose ${ }^{49}$ utilized an even higher concentration, viz., 22\%. Herein we used a lipid concentration of $8 \%$ and efforts were made to increase the order of the system, thus raising the magnitude of the RDCs, by increasing the lipid concentration (to $12 \%$ ). These attempts, however, were fruitless as an appearance, in the deuterium NMR spectrum, of an isotropic (central) peak in addition to the quadrupolar coupling of $\mathrm{D}_{2} \mathrm{O}$ indicated decreased stability of the sample. The physicochemical properties of sucrose and TRH are undeniably different, ${ }^{78,79}$ even though they are both nonreducing hexose-containing disaccharides, and thus, what appears to be small changes in the molecular structure, are indeed responsible for the experimental difficulties in studying TRH, as compared to sucrose.

In order to derive the information about molecular structure from the experimental and simulated dipolar couplings eqn (1) is transformed into the following relationship ${ }^{80}$

$$
\begin{aligned}
d_{i j}= & -\frac{\mu_{0}}{16 \pi^{2}} \frac{\gamma_{i} \gamma_{j} \hbar}{r_{i j}{ }^{3}} \\
& \times\left[S_{z z}\left(3 \cos ^{2} \theta_{i j}^{z}-1\right)+\left(S_{x x}-S_{y y}\right)\left(\cos ^{2} \theta_{i j}^{x}-\cos ^{2} \theta_{i j}^{y}\right)\right. \\
& \left.+4 S_{x y} \cos \theta_{i j}^{x} \cos \theta_{i j}^{y}+4 S_{x z} \cos \theta_{i j}^{x} \cos \theta_{i j}^{z}+4 S_{y z} \cos \theta_{i j}^{y} \cos \theta_{i j}^{z}\right]
\end{aligned}
$$

where $\theta_{i j}^{a}(a=x, y, z)$ are angles between the spin-spin vector and the molecular-fixed axes indicated in Fig. 1. The elements of the Saupe matrix $\boldsymbol{S}$, given by $S_{a b}(a, b=x, y, z)$, are called the order parameters. If the dipolar coupling (eqn (2)) occurs between spins in different molecular fragments, $r_{i j}, S_{a b}$, and $\theta_{i j}^{a}$, become dependent on the molecular structure. The directional cosines were taken as averages derived from the trajectory generated in the MD computer simulations and the order parameters were used as fitting parameters. The TRH molecule possesses $C_{2}$ symmetry; therefore, only three order parameters $S_{z z}, S_{x x}-S_{y y}$ and $S_{x y}$ are required for the full description of the orientational order. The molecular coordinate system was chosen (see Fig. 1) so that the $C_{2}$-axis of the trehalose molecule coincides with the $z$-axis, the $x$-axis is parallel to the vector between the $\mathrm{C} 1$ and $\mathrm{C}^{\prime}$ atoms and the $y$ direction is orthogonal to the $z$ - and $x$-axes.

The experimental couplings, $d_{i j}^{\text {expt }}$, are displayed in Fig. 5A. In the quantitative analysis, we employed a numerical fitting procedure of all the couplings using eqn (2) with the three relevant elements of the order tensor $\left(S_{z z}, S_{x x}-S_{y y}, S_{x y}\right)$ as the fitting parameters. The fitting was performed using a computer code (Octave, version 3.0, http://www.gnu.org), ${ }^{81}$ which minimizes the error sum, $Q$

$$
Q=\sqrt{\frac{1}{n} \sum_{i j}\left(d_{i j}^{\text {expt }}-d_{i j}^{\text {calc }}\right)^{2}}
$$

where $n$ is the number of dipolar couplings used in the analysis, whereas $d_{i j}^{\text {expt }}$ and $d_{i j}^{\text {calc }}$ are the experimental and calculated couplings, respectively. The indicated experimental errors are $\pm 0.2 \mathrm{~Hz}$, which are based on repeated as well as different experiments.

In order to include the uncertainties of conformations an error analysis of the back-calculated dipolar couplings, $d_{i j}^{\text {calc }}$, was carried out by calculating normal distributions of $d_{i j}^{\text {expt }}$. In practice, 10000 random sets of dipolar couplings were 


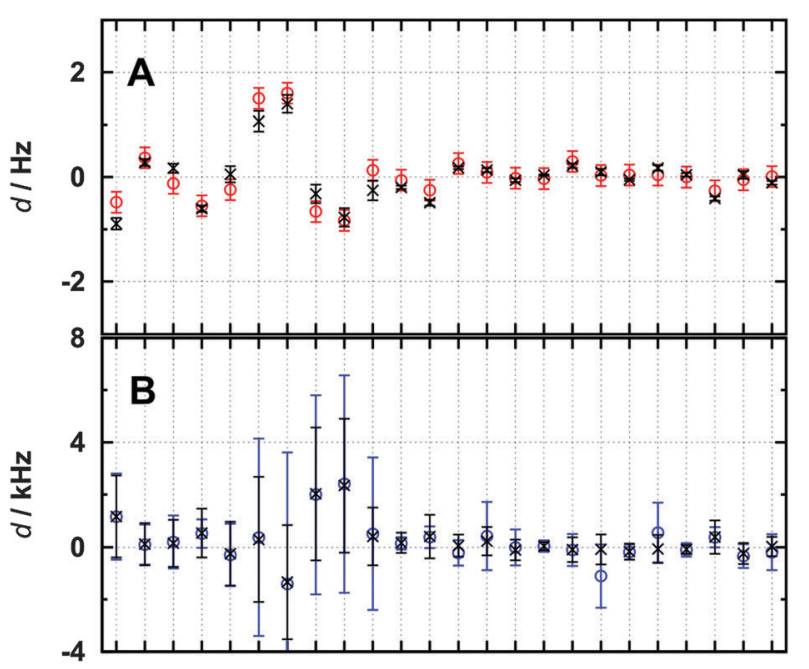

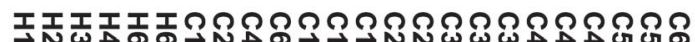

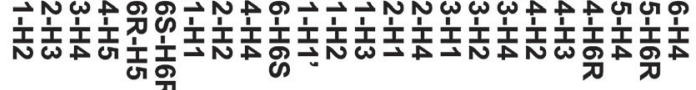

Fig. 5 Experimental NMR dipolar couplings in TRH (red symbols) from the dilute lyotropic (DMPC + DHPC) liquid crystal (A) and derived from the trajectory generated in an MD simulation of the TRH2O (blue symbols) sample (B). For the calculated values, represented by black symbols with associated error bars, the order parameters $S_{z z}=(-1.84 \pm 0.26) \times 10^{-4}$, $S_{x x}-S_{y y}=(0.88 \pm 0.20) \times 10^{-4}$, and $S_{x y}=(-2.6 \pm 3.1) \times 10^{-5}$ were used (A), and $S_{z z}=0.03 \pm 0.4, S_{x x}-S_{y y}=0.31 \pm 0.32$ and $S_{x y}=0.01 \pm 0.31$ (B). These order parameters are averages obtained from the fitting procedure containing 10000 cycles, which is described in the text.

generated by using averaged values of $d_{i j}^{\text {expt }}$ and the corresponding errors. To ensure the generation of realistic dipolar couplings, the normal distribution was truncated at $\pm 3 \sigma$. The five relevant cosines in eqn (2) were determined from the trajectory together with their standard deviations, $\sigma_{\cos \theta_{i j}}^{\operatorname{sim}}$

$$
\sigma_{\cos \theta_{i j}}^{\operatorname{sim}}=\left\langle\left(\left\langle\cos \theta_{i j}^{\operatorname{sim}}\right\rangle_{t}-\left\langle\cos \theta_{i j}^{\operatorname{sim}}\right\rangle_{N, t}\right)^{2}\right\rangle_{N}^{1 / 2},
$$

where $N$ is the number of TRH molecules and $t$ is the time (length of the trajectory).

Again, 10000 normally distributed cosine-values were generated in the interval $\pm 3 \sigma_{\cos \theta_{i j}}^{\operatorname{sim}}$. Finally, the data set of $d_{i j}^{\operatorname{expt}}$ was used together with the cosine-values to fit the order tensors. This procedure resulted in a distribution of back-calculated dipolar couplings that were used to calculate an average and a standard deviation for each coupling, $d_{i j}^{\text {calc }}$, corresponding to $d_{i j}^{\text {expt }}$. The agreement between the experimental and back-calculated dipolar couplings (Fig. 5A) is very good. The analysis is however clearly limited by the very small magnitude of experimental dipolar couplings: in fact many couplings are smaller than the experimental errors.

We have also calculated dipolar couplings using eqn (1) and the TRH20 MD trajectory. These couplings, $d_{i j}^{\text {calc }}$, were analyzed employing the same procedure as for the experimental couplings above, and the results are collected in Fig. 5B. We note that the simulated couplings are three orders of magnitude larger compared with the experimentally determined counterparts. The reason is that in the MD simulations the TRH molecules spend most of the time attached to the surface rather than as free molecules in the bulk water phase. The composition of the experimental sample is approximately $1: 2.5: 1100$ (50:127:55000 mM) for trehalose, lipids and water, whereas the corresponding ratio in the MD simulation box in the TRH20 system is $1: 6.4: 500(20: 128: 10000$ given as the number of molecules). Clearly, the real sample contains a larger water bulk and smaller membrane surface per TRH molecule, which may partly explain a weaker association. Other possible sources of the dramatic difference between the experimental and simulated dipolar couplings are associated with a short simulation time (140 ns) that prevents efficient averaging of the couplings, and in addition, a too attractive interaction potential. ${ }^{82}$

\section{Molecular conformations}

In Fig. 6 the conformational distribution functions are displayed for TRH with different lipid concentrations calculated from the MD trajectories for the three relevant torsion angles $\phi$, $\omega$ and $\theta$ in the molecule, corresponding to $\mathrm{H} 1-\mathrm{C} 1-\mathrm{O} 1-\mathrm{C}^{\prime}$, O5-C5-C6-O6 and C5-C6-O6-HO6, respectively (cf. Fig. 1). Clearly, there is no significant TRH concentration dependence for any of the torsion angles under the different conditions employed.
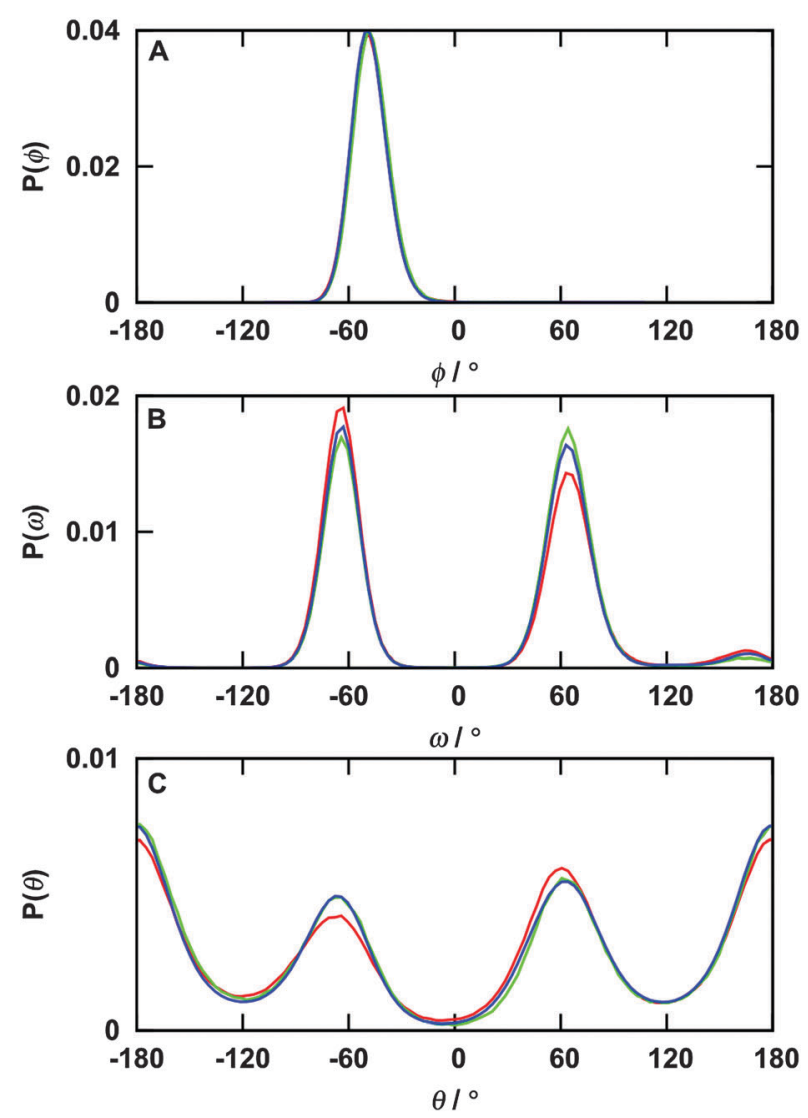

Fig. 6 Probability distribution functions, derived from the MD trajectory, for the torsion angles $\phi(\mathrm{A}), \omega(\mathrm{B})$ and $\theta$ (C) in trehalose, see Fig. 1 for three different samples: TRH50-ISO (green) in the isotropic solution, TRH2O (red), and $\mathrm{TRH} 230$ (blue) in the ordered phase. 
The distribution functions determined for different concentrations of TRH in the isotropic phase are essentially identical (not shown). The $\phi$ torsion angle distribution exhibits, as expected, only one state with $\langle\phi\rangle=-47.2^{\circ}$. Using a Karplus-type relationship $^{83}$ an average $J$ coupling for $\mathrm{H} 1-\mathrm{C}^{\prime}$ of $3.54 \pm 0.17 \mathrm{~Hz}$ was calculated from the TRH20 distribution in Fig. 6A, and $3.62 \pm$ $0.06 \mathrm{~Hz}$ in the isotropic phase. The corresponding experimental value was determined to be $3.31 \pm 0.25 \mathrm{~Hz}$, in good agreement with literature data of $3.0 \pm 0.3^{25}$ and $3.3 \pm 0.5 \mathrm{~Hz} .{ }^{84}$ Thus, the heteronuclear $J$ value from the MD simulation agrees well with that of the experiment, for which the error has been estimated by the developers of the NMR experiment. ${ }^{61}$ Furthermore, the interresidue conformationally averaged effective proton-proton distances can be compared with experimentally determined ${ }^{1} \mathrm{H},{ }^{1} \mathrm{H}$ NOE data. Using an $\left\langle r^{-6}\right\rangle$-relationship the effective distances derived from the MD trajectory of an isotropic solution resulted in $\left\langle r_{\mathrm{H} 1, \mathrm{H} 1^{\prime}}^{-6}\right\rangle^{-1 / 6}=2.66 \pm 0.02$ and $\left\langle r_{\mathrm{H} 1, \mathrm{H} 5^{\prime}}^{-6}\right\rangle^{-1 / 6}=3.00 \pm 0.02 \AA$ for $\mathrm{H} 1-\mathrm{H} 1^{\prime}$ and $\mathrm{H} 1-\mathrm{H}^{\prime}$, respectively. The effective distances in the ordered TRH20 sample were essentially identical: $\left\langle r_{\mathrm{H} 1, \mathrm{H} 1^{\prime}}^{-6}\right\rangle^{-1 / 6}=$ $2.68 \pm 0.02$ and $\left\langle r_{\mathrm{H} 1, \mathrm{H}^{\prime}}^{-6}\right\rangle^{-1 / 6}=2.96 \pm 0.02 \AA$. In a study of trehalose based on molecular mechanics and NOE data ${ }^{25}$ the corresponding distances were 2.8 and $2.5 \AA$. Thus, the effective $\mathrm{H} 1-\mathrm{H} 1^{\prime}$ inter-residue distance agrees reasonably well with the corresponding one derived from the present MD simulation, whereas for the $\mathrm{H} 1-\mathrm{H} 5$ ' distance in experiment and simulations disagree significantly. It can, however, be noted that the estimated error in the experimental $\mathrm{H} 1-\mathrm{H}^{\prime}$ distance is very large $( \pm 2.0 \AA) .{ }^{25}$ The $\omega$ distributions displayed in Fig. 6B consist of three conformational states: $+g\left(\omega=+65^{\circ}\right),-g\left(\omega=-65^{\circ}\right)$, and $t\left(\omega=180^{\circ}\right)$, where $g$ and $t$ denote the gauche and trans relationships for O5 and O6. Populations of the three conformational states related to the $\omega$ torsion angle were determined by integrating the MD probability distribution functions in Fig. 6B. The populations derived from the different MD trajectories are collected in Table 2, being similar in both the ordered and isotropic phases (distributions not shown here). The populations derived from the experimental spin-spin couplings in the isotropic phase were obtained by fitting ${ }^{3} J_{\mathrm{H} 5, \mathrm{H} 6 \text { pro- } R}=5.25 \mathrm{~Hz}$ and ${ }^{3} J_{\mathrm{H} 5 \text {,H6pro- } S}=2.38 \mathrm{~Hz}$ using the $+g,-g$ and $t$ populations

Table 2 Population distributions of the $\omega$ torsion angle in TRH determined from NMR and predicted from different MD simulations based on ${ }^{3} J_{\text {H5,H6pro-R }}$ and ${ }^{3} J_{\text {H5,H6pro-S }}$ values. The distribution for $\omega$ in $\alpha$-D-Glcp-OMe is also included

\begin{tabular}{llllll}
\hline Method & $g\left(-60^{\circ}\right)$ & $g\left(60^{\circ}\right)$ & $t\left(180^{\circ}\right)$ & ${ }^{3} J_{\mathrm{H} 5, \mathrm{H} 6 \text { pro- } R}$ & $3_{\mathrm{H} 5, \mathrm{H} 6 \text { pro- } S}$ \\
\hline NMR $^{3}$ & 0.47 & 0.43 & 0.10 & 5.25 & 2.38 \\
TRH10-ISO $^{a}$ & 0.44 & 0.53 & 0.03 & $5.54 \pm 0.62$ & $1.962 \pm 0.052$ \\
TRH50-ISO $_{\text {TRH100-ISO }}$ & 0.46 & 0.51 & 0.03 & $5.46 \pm 1.62$ & $1.98 \pm 0.42$ \\
TRH20-128DMPC $^{b}$ & 0.44 & 0.52 & 0.04 & $5.36 \pm 1.89$ & $2.04 \pm 0.43$ \\
TRH80-128DMPC $^{2}$ & 0.45 & 0.45 & 0.05 & $4.87 \pm 1.17$ & $2.03 \pm 0.41$ \\
NMR $(\alpha-\mathrm{D}-G l c p-O M e)^{0.49}$ & 0.42 & 0.04 & $5.40 \pm 1.67$ & $2.02 \pm 0.50$ \\
\end{tabular}

${ }^{a}$ TRH10-ISO: MD simulation of an isotropic phase -10 TRH molecules.

${ }^{b}$ TRH20-128DMPC: MD simulation of a lipid bilayer -20 TRH molecules. as fitting parameters. We have also determined the corresponding $J$ couplings in $\alpha$-D-Glcp-OMe, ${ }^{3} J_{\mathrm{H} 5, \mathrm{H} 6 \text { pro- } R}=5.58 \mathrm{~Hz}$ and ${ }^{3} J_{\mathrm{H} 5, \mathrm{H} 6 \text { pro- } S}=2.28 \mathrm{~Hz}$, thus being closely similar and consequently so are the $\omega$ torsion angle distributions (Table 2). The relationship between the $J$ coupling and the conformational state (torsion angle) was established employing a Karplus-type equation. ${ }^{85}$ The corresponding couplings derived from the TRH20 MD trajectory were ${ }^{3} J_{\mathrm{H} 5, \mathrm{H} 6 \text { pro- } R}=4.9 \pm 1.2 \mathrm{~Hz}$ and ${ }^{3} J_{\mathrm{H} 5, \mathrm{H} 6 \mathrm{pro}-\mathrm{S}}=2.03 \pm 0.41 \mathrm{~Hz}$.

We also consider the conformational transitions related to the $\theta$ torsion angle. The probability distributions are displayed in Fig. 6C. Here all three conformational states are significantly populated, the trans conformation being the major one and the two gauche conformations are approximately equally populated. Given that the agreement between the experiment and simulation is very good for the $\omega$ torsion angle in TRH, we set out to evaluate a relationship, which also includes the $\theta$ torsion angle. The ${ }^{2} J_{\text {H6pro- } R \text {,H6pro- } S}$ coupling constant is dependent on the conformational distributions at the $\omega$ and $\theta$ torsion angles and was calculated from the MD trajectories based on two different Karplus-type relationships

$$
\begin{aligned}
{ }^{2} J_{\text {H6pro- } R, \text { H6pro- } S}= & -11.26+0.76 \cos (2 \omega)+2.02 \cos (2 \theta) \\
{ }^{2} J_{\text {H6pro- } R, \text { H6pro- } S}= & -11.23+0.13 \cos (\omega)+0.74 \cos (2 \omega) \\
& -0.82 \cos (\theta)+2.02 \cos (2 \theta)
\end{aligned}
$$

where the former ${ }^{85}$ having three terms subsequently was extended to a five term version. ${ }^{86}$ The experimental value in the isotropic solution is given by ${ }^{2} J_{\mathrm{H} 6 \text { pro- } R \text {, H6pro- } S}=-12.08 \mathrm{~Hz}$, and the values calculated from the MD simulation of an isotropic phase using eqn (5) and (6), were $-10.924 \pm 0.030$ and $-11.373 \pm 0.043 \mathrm{~Hz}$, respectively. Using the TRH20 trajectory the corresponding values of ${ }^{2} J_{\mathrm{H} 6 \text { pro- } R \text {, H6pro- } S}$ obtained employing eqn (5) and (6) were $-10.96 \pm 0.26$ and $-11.41 \pm 0.35 \mathrm{~Hz}$, respectively. The difference between experimental and calculated couplings is within $\sim 1 \mathrm{~Hz}$ and the higher magnitude of the experimentally determined value suggests that gauche conformations are present to a larger extent than what is observed from the MD simulations. Furthermore, the calculated $J$ couplings in the ordered phase and the isotropic solution are essentially identical.

\section{Bilayer-trehalose interactions}

Solvation and hydrogen bonding. Radial distribution functions (RDFs) for hydroxyl hydrogens in trehalose and phosphate oxygens in DMPC are displayed in Fig. 7A-C for TRH20, TRH80 and TRH230. The high intensity of all (except HO6) the RDFs indicate a solid-like hydrogen bond structure with a low degree of motion or exchange. The intensity decreases with increased concentration of TRH reflecting the fact that for a larger number of TRHs a smaller fraction is involved in hydrogen bonds with DMPC. The HO3 hydrogen atom in TRH is the most effective participant in the formation of hydrogen bonds with DMPC followed by $\mathrm{HO} 2, \mathrm{HO} 4$ and $\mathrm{HO}$. In principle, it can be expected that $\mathrm{HO} 3$ and $\mathrm{HO} 4$ are equally exposed to interactions with lipids. The phosphate group of the lipids $\mathrm{OP}_{\mathrm{pro}-R}$ and $\mathrm{OP}_{\mathrm{pro}-S}$ are 

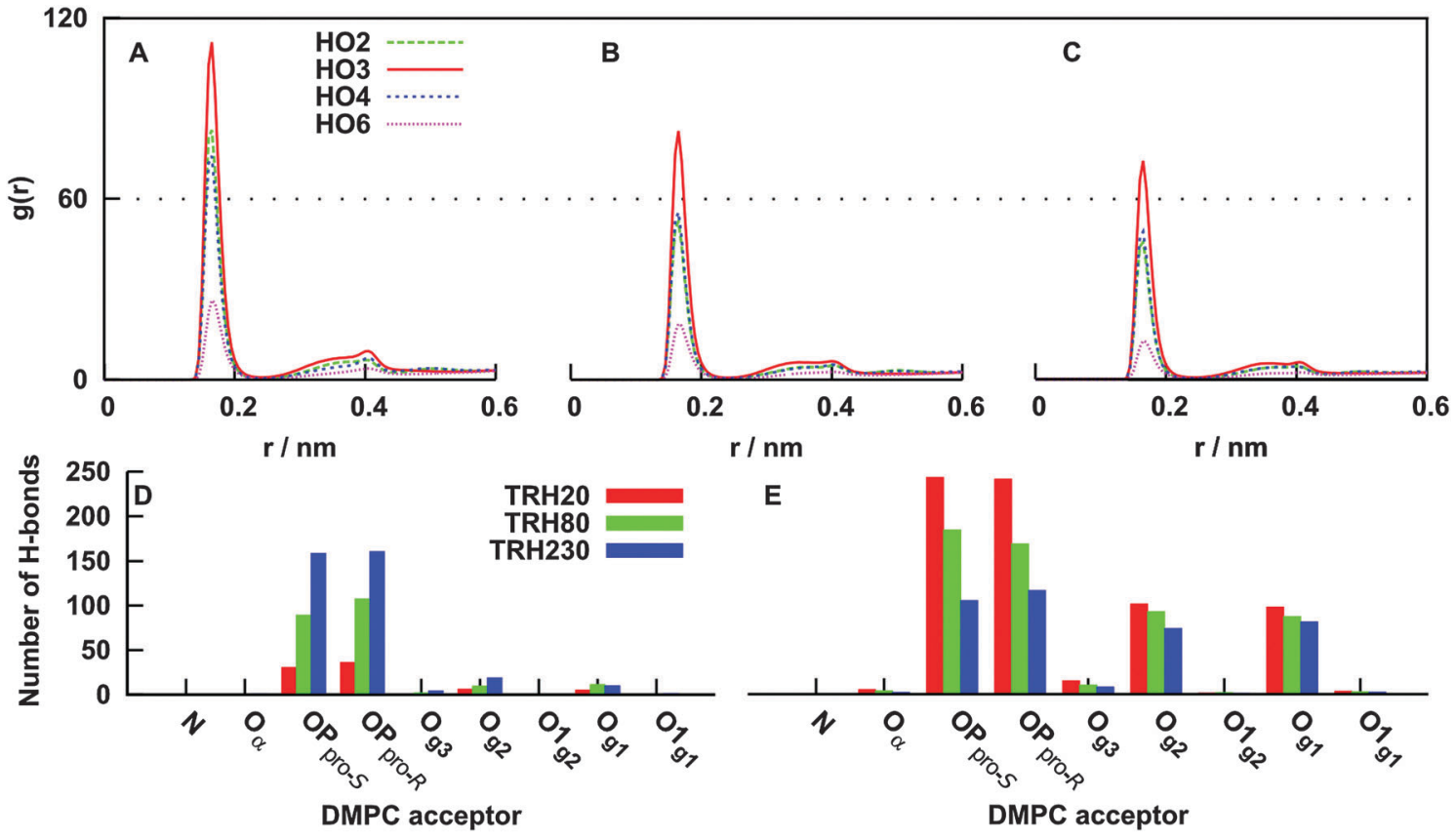

Fig. 7 Radial distribution functions (RDFs) calculated for hydrogen atoms in TRH and phosphate oxygens in DMPC: TRH20 (A), TRH80 (B) and TRH230 (C). The average number of hydrogen bonds at every time step for DMPC with TRH (D) and water (E).

accessible for hydrogen bond formation with TRH and water (Fig. 7D and E). These two sites are the only hydrogen bond acceptors for TRH, whereas $\mathrm{O}_{\mathrm{g} 1}$ and $\mathrm{O}_{\mathrm{g} 2}$ readily interact with water.
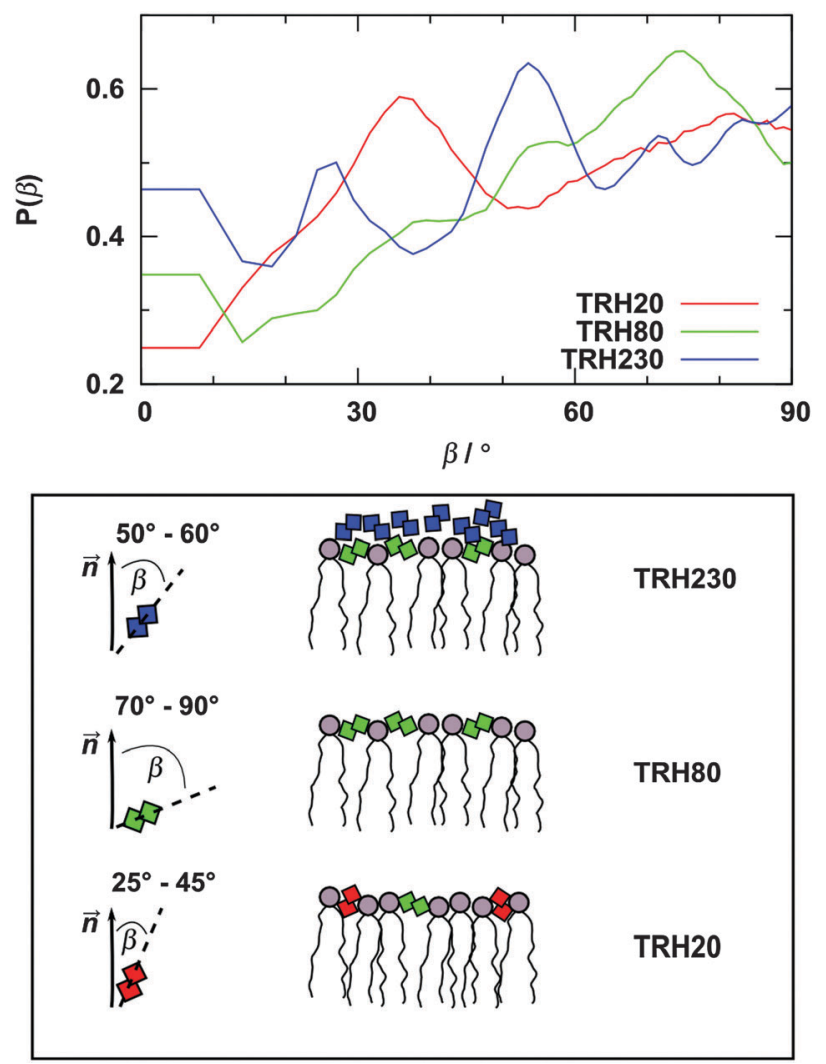

Fig. 8 The orientational distribution functions $P(\beta)$ for the $\mathrm{C} 4-\mathrm{C} 4^{\prime}$ vector in TRH relative to the bilayer normal.
An increased number of trehalose molecules leads to an increased total number of DMPC-TRH hydrogen bonds, whereas a decreased number of DMPC-water interactions are observed. This finding is clearly consistent with one of the hypotheses for trehalosemembrane interaction where water expulsion from the interface is assumed.

In Fig. 8 the orientational probability distributions, $P(\beta)$, are displayed for the $\mathrm{C} 4-\mathrm{C} 4{ }^{\prime}$ vector in trehalose relative to the bilayer normal. The TRH20 distribution exhibits one major relatively narrow maximum at $\beta=35^{\circ}$, which may reflect a single hydrogen bond of the hydroxyl group at the C3 or C4 atoms of TRH to a phosphate oxygen atom. It can be speculated that the high population of large angles, $>60^{\circ}$, is related to TRH molecules that form hydrogen bonds with two different lipids, for which the $\mathrm{C} 4-\mathrm{C} 4{ }^{\prime}$ vector has a perpendicular orientation relative to the bilayer normal, i.e. $\beta \approx 90^{\circ}$. Upon increased TRH content, the maximum of $P(\beta)$ shifts to larger angles. At an even higher concentration, in the TRH230 sample, several distinct orientations are present where the TRH molecules interact with both DMPC lipids and associate with each other.

\section{Conclusions}

By NMR spectroscopy and molecular dynamics computer simulations the lipid membrane-trehalose interactions and conformations of the TRH molecule have been investigated. Both the previously reported MD simulations ${ }^{5}$ and the NMR experiments were carried out in the isotropic solution and in the ordered phase. NMR studies in weakly ordered media facilitate the acquisition of both hetero- and homonuclear residual dipolar couplings (RDCs), which provide important information about the molecular conformations and the orientational order. 
One of the major assumptions in the interpretations of RDC, collected in weakly ordered liquid crystals, is that the molecular conformations in the isotropic liquid and in the ordered phase are similar. Combination of the NMR experiments and MD simulations is a powerful tool to test the validity of this assumption. The RDCs in trehalose calculated from the MD trajectory were three orders of magnitude larger compared with the experimentally determined counterparts. The reason for this dramatic discrepancy has been attributed to: (a) a relatively short simulation time, (b) slow exchange of TRH molecules between the membrane surface and the water bulk, and (c) slightly smaller water bulk and larger membrane surface per TRH molecule in the MD simulation. There was, however, good agreement between the experimental and the MD trajectory calculated scalar $J$ couplings, which indicates a correct description of molecular conformations in the MD simulation. Thus, although the orientational order in the MD simulation was not adequately described, the conformations were, and these were subsequently used in the interpretation of the experimental RDCs. It should, however, be noted that the error of the orientational order is given by $\sigma(S) \sim 1 / \sqrt{N}$, where $N$ is the number of molecules in the MD simulation. Thus, for TRH20 the error is $\sim 0.2$, significantly larger than the experimental order parameter. The molecular conformations in the isotropic and ordered phases were highly similar which, as mentioned above, is the major assumption in all the analyses of RDCs.

Finally, from the MD trajectory we have calculated one-bond C-H NMR dipolar couplings in the DMPC lipids, and compared these with experimental values previously determined ${ }^{42}$ by solidstate NMR spectroscopy. The agreement between calculated and experimental couplings is very good, except for the glycerol fragment, which depends on the poor description of the torsional potential of this fragment. The dipolar couplings calculated from the MD trajectory also include the sign of the interaction, which is not available from the solid-state NMR experiments.

\section{Acknowledgements}

This work was supported by grants from the Swedish Research Council, the Knut and Alice Wallenberg Foundation, the Carl Trygger Foundation and the Swedish National Infrastructure for Computing (SNAC 001-12-102) via PDC (www.pdc.kth.se).

\section{References}

1 H. D. Andersen, C. Wang, L. Arleth, G. H. Peters and P. Westh, Proc. Natl. Acad. Sci. U. S. A., 2011, 108, 1874-1878.

2 C. W. Lee, S. K. Das Gupta, J. Mattai, G. G. Shipley, O. H. Abdel-Mageed, A. Makriyannis and R. G. Griffin, Biochemistry, 1989, 28, 5000-5009.

3 B. A. Brüning, S. Prévost, R. Stehle, R. Steitz, P. Falus, B. Farago and T. Hellweg, Biochim. Biophys. Acta, Biomembr., 2014, 1838, 2412-2419.

4 J. Tian, A. Sethi, B. I. Swanson, B. Goldstein and S. Gnanakaran, Biophys. J., 2013, 104, 622-632.
5 J. Kapla, J. Wohlert, B. Stevensson, O. Engström, G. Widmalm and A. Maliniak, J. Phys. Chem. B, 2013, 117, 6667-6673.

6 J. H. Crowe, L. M. Crowe and D. Chapman, Science, 1984, 223, 701-703.

7 L. M. Crowe, R. Mouradian, J. H. Crowe, S. A. Jackson and C. Womersley, Biochim. Biophys. Acta, 1984, 769, 141-150.

8 E. A. Golovina, A. V. Golovin, F. A. Hoekstra and R. Faller, Biophys. J., 2009, 97, 490-499.

9 M. Nakagaki, H. Nagase and H. Ueda, J. Membr. Sci., 1992, 73, 173-180.

10 G. Cottone, G. Ciccotti and L. Cordone, J. Chem. Phys., 2002, 117, 9862-9866.

11 R. D. Lins, C. S. Pereira and P. H. Hünenberger, Proteins, 2004, 55, 177-186.

12 P. S. Belton and A. M. Gil, Biopolymers, 1994, 34, 957-961.

13 C. Lambruschini, A. Relini, A. Ridi, L. Cordone and A. Gliozzi, Langmuir, 2000, 16, 5467-5470.

14 L. Cordone, G. Cottone, S. Giuffrida, G. Palazzo, G. Venturoli and C. Viappiani, Biochim. Biophys. Acta, Proteins Proteomics, 2005, 1749, 252-281.

15 A. M. Massari, I. J. Finkelstein, B. L. McClain, A. Goj, X. Wen, K. L. Bren, R. F. Loring and M. D. Fayer, J. Am. Chem. Soc., 2005, 127, 14279-14289.

16 W. Q. Sun and A. C. Leopold, Comp. Biochem. Physiol., Part A: Mol. Integr. Physiol., 1997, 117, 327-333.

17 K. L. Koster, M. S. Webb, G. Bryant and D. V. Lynch, Biochim. Biophys. Acta, Biomembr., 1994, 1193, 143-150.

18 K. L. Koster, Y. P. Lei, M. Anderson, S. Martin and G. Bryant, Biophys. J., 2000, 78, 1932-1946.

19 W. Q. Sun, A. C. Leopold, L. M. Crowe and J. H. Crowe, Biophys. J., 1996, 70, 1769-1776.

20 W. Q. Sun and A. C. Leopold, Ann. Bot., 1994, 74, 601-604.

21 L. R. Winther, J. Qvist and B. Halle, J. Phys. Chem. B, 2012, 116, 9196-9207.

22 L. Lupi, L. Comez, M. Paolantoni, S. Perticaroli, P. Sassi, A. Morresi, B. M. Ladanyi and D. Fioretto, J. Phys. Chem. B, 2012, 116, 14760-14767.

23 M. Heyden, G. Schwaab and M. Havenith, J. Phys. Chem. B, 2014, 118, 10802-10805.

24 B. Halle, J. Phys. Chem. B, 2014, 118, 10806-10812.

25 A. Poveda, C. Vicent, S. Penadés and J. Jiménez-Barbero, Carbohydr. Res., 1997, 301, 5-10.

26 A. D. French, G. P. Johnson, A. M. Kelterer, M. K. Dowd and C. J. Cramer, J. Phys. Chem. A, 2002, 106, 4988-4997.

27 M. M. Kuttel and K. J. Naidoo, Carbohydr. Res., 2005, 340, 875-879.

28 A. Skibinsky, R. M. Venable and R. W. Pastor, Biophys. J., 2005, 89, 4111-4121.

29 C. S. Pereira and P. H. Hünenberger, Biophys. J., 2008, 95, 3525-3534.

30 A. K. Sum, R. Faller and J. J. de Pablo, Biophys. J., 2003, 85, 2830-2844.

31 S. Leekumjorn and A. K. Sum, Mol. Simul., 2006, 32, 219-230.

32 M. A. Villarreal, S. B. Díaz, E. A. Disalvo and G. G. Montich, Langmuir, 2004, 20, 7844-7851. 
33 C. S. Pereira, R. D. Lins, I. Chandrasekhar, L. C. G. Freitas and P. H. Hünenberger, Biophys. J., 2004, 86, 2273-2285.

34 C. S. Pereira and P. H. Hünenberger, Mol. Simul., 2008, 34, 403-420.

35 G. Moiset, C. A. López, R. Bartelds, L. Syga, E. Rijpkema, A. Cukkemane, M. Baldus, B. Poolman and S. J. Marrink, J. Am. Chem. Soc., 2014, 136, 16167-16175.

36 L. Sapir and D. Harries, J. Phys. Chem. B, 2011, 115, 624-634.

37 A. Bax and N. Tjandra, J. Biomol. NMR, 1997, 10, 289-292.

38 N. Tjandra and A. Bax, Science, 1997, 278, 1111-1114.

39 J. H. Prestegard, H. M. Al-Hashimi and J. R. Tolman, Q. Rev. Biophys., 2000, 33, 371-424.

40 J. D. Gross, D. E. Warschawski and R. G. Griffin, J. Am. Chem. Soc., 1997, 119, 796-802.

41 D. McElheny, E. DeVita and L. Frydman, J. Magn. Reson., 2000, 143, 321-328.

42 V. Castro, B. Stevensson, S. V. Dvinskikh, C. J. Högberg, A. P. Lyubartsev, H. Zimmermann, D. Sandström and A. Maliniak, Biochim. Biophys. Acta, Biomembr., 2008, 1778, 2604-2611.

43 S. V. Dvinskikh, D. Sandström, Z. Luz, H. Zimmermann and A. Maliniak, J. Chem. Phys., 2003, 119, 413.

44 H. Neubauer, J. Meiler, W. Peti and C. Griesinger, Helv. Chim. Acta, 2001, 84, 243-258.

45 C. Landersjö, B. Stevensson, R. Eklund, J. Östervall, P. Söderman, G. Widmalm and A. Maliniak, J. Biomol. NMR, 2006, 35, 89-101.

46 B. Stevensson, C. Landersjö, G. Widmalm and A. Maliniak, J. Am. Chem. Soc., 2002, 124, 5946-5947.

47 M. Staaf, C. Höög, B. Stevensson, A. Maliniak and G. Widmalm, Biochemistry, 2001, 40, 3623-3628.

48 A. Almond, J. Bunkenborg, T. Franch, C. H. Gotfredsen and J. Duus, J. Am. Chem. Soc., 2001, 123, 4792-4802.

49 D. I. Freedberg, J. Am. Chem. Soc., 2002, 124, 2358-2362.

50 X. Yi, A. Venot, J. Glushka and J. H. Prestegard, J. Am. Chem. Soc., 2004, 126, 13636-13638.

51 G. Nodet, L. Poggi, D. Abergel, C. Gourmala, D. Dong, Y. Zhang, J. M. Mallet and G. Bodenhausen, J. Am. Chem. Soc., 2007, 129, 9080-9085.

52 J. P. M. Jämbeck and A. P. Lyubartsev, J. Phys. Chem. B, 2012, 116, 3164-3179.

53 K. N. Kirschner, A. B. Yongye, S. M. Tschampel, J. GonzálezOuteiriño, C. R. Daniels, B. L. Foley and R. J. Woods, J. Comput. Chem., 2008, 29, 622-655.

54 W. L. Jorgensen, J. Chandrasekhar, J. D. Madura, R. W. Impey and M. L. Klein, J. Chem. Phys., 1983, 79, 926-935.

55 D. J. Price and C. L. Brooks, J. Chem. Phys., 2004, 121, 10096. 56 S.-T. Lin, P. K. Maiti and W. A. Goddard, J. Phys. Chem. B, 2010, 114, 8191-8198.

57 B. Hess, C. Kutzner, D. van der Spoel and E. Lindahl, J. Chem. Theory Comput., 2008, 4, 435-447.

58 U. Essmann, L. Perera, M. L. Berkowitz, T. Darden, H. Lee and L. G. Pedersen, J. Chem. Phys., 1995, 103, 8577-8593.

59 J. Rönnols, R. Pendrill, C. Fontana, C. Hamark, T. Angles d'Ortoli, O. Engström, J. Ståhle, M. V. Zaccheus, E. Säwén,
L. E. Hahn, S. Iqbal and G. Widmalm, Carbohydr. Res., 2013, 380, 156-166.

60 M. Findeisen, T. Brand and S. Berger, Magn. Reson. Chem., 2007, 45, 175-178.

61 A. Meissner and O. W. Sørensen, Magn. Reson. Chem., 2001, 39, 49-52.

62 W. Koźmiński and D. Nanz, J. Magn. Reson., 1997, 124, 383-392.

63 W. Kozmiński and D. Nanz, J. Magn. Reson., 2000, 142, 294-299.

64 F. Tian, H. M. Al-Hashimi, J. L. Craighead and J. H. Prestegard, J. Am. Chem. Soc., 2001, 123, 485-492.

65 A. E. Derome and M. P. Williamson, J. Magn. Reson., 1990, 88, 177-185.

66 F. del Río-Portilla, E. Sánchez-Mendoza, V. U. ConstantinoCastillo and J. A. del Río, ARKIVOC, 2003, xi, 213-226.

67 V. Castro, S. V. Dvinskikh, G. Widmalm, D. Sandström and A. Maliniak, Biochim. Biophys. Acta, Biomembr., 2007, 1768, 2432-2437.

68 B. Hess, H. Bekker, H. J. C. Berendsen and J. G. E. M. Fraaije, J. Comput. Chem., 1997, 18, 1463-1472.

69 B. Hess, J. Chem. Theory Comput., 2008, 4, 116-122.

70 E. R. Henry and A. Szabo, J. Chem. Phys., 1985, 82, 4753-4761.

71 O. H. S. Ollila, 2013, arXiv:1309.2131v2 [physics.bio-ph], 7.

72 C. Zannoni and M. Guerra, Mol. Phys., 1981, 44, 849-869.

73 A. Maliniak and G. Widmalm, in Food Oligosaccharides: Production, Analysis and Bioactivity, ed. F. Javier Moreno and M. Luz Sanz, John Wiley \& Sons, Ltd., 2014, pp. 320-349.

74 K. Lycknert, A. Maliniak and G. Widmalm, J. Phys. Chem. A, 2001, 105, 5119-5122.

75 N. G. A. Bell, G. Rigg, S. Masters, J. Bella and D. Uhrín, Phys. Chem. Chem. Phys., 2013, 15, 18223-18234.

76 T. Rundlöf, C. Landersjö, K. Lycknert, A. Maliniak and G. Widmalm, Magn. Reson. Chem., 1998, 36, 773-776.

77 C. Landersjö, C. Höög, A. Maliniak and G. Widmalm, J. Phys. Chem. B, 2000, 104, 5618-5624.

78 M. D. Battistel, R. Pendrill, G. Widmalm and D. I. Freedberg, J. Phys. Chem. B, 2013, 117, 4860-4869.

79 K. Shiraga, T. Suzuki, N. Kondo, J. De Baerdemaeker and Y. Ogawa, Carbohydr. Res., 2015, 406, 46-54.

80 R. Y. Dong, Nuclear Magnetic Resonance of Liquid Crystals, Springer-Verlag, New York, 1994.

$81 \mathrm{~J}$. W. Eaton, D. Bateman and S. Hauberg, GNU Octave Manual; Version 3, Network Theory Ltd, 2008.

82 E. Hatcher, E. Säwén, G. Widmalm and A. D. MacKerell Jr., J. Phys. Chem. B, 2011, 115, 597-608.

83 E. Säwén, T. Massad, C. Landersjö, P. Damberg and G. Widmalm, Org. Biomol. Chem., 2010, 8, 3684-3695.

84 G. Batta, K. E. Kövér, J. Gervay, M. Hornyák and G. M. Roberts, J. Am. Chem. Soc., 1997, 119, 1336-1345.

85 R. Stenutz, I. Carmichael, G. Widmalm and A. S. Serianni, J. Org. Chem., 2002, 67, 949-958.

86 C. Thibaudeau, R. Stenutz, B. Hertz, T. Klepach, S. Zhao, Q. Wu, I. Carmichael and A. S. Serianni, J. Am. Chem. Soc., 2004, 126, 15668-15685. 